Proceedings of the ASME 2012 31st International Conference on Ocean, Offshore and Arctic Engineering
OMAE2012

June 10-15, 2012, Rio de Janeiro, Brazil

OMAE2012-83760

\title{
AN EFFICIENT 3-D FNPF NUMERICAL WAVE TANK FOR VIRTUAL LARGE-SCALE WAVE BASIN EXPERIMENT
}

\author{
Seshu Nimmala, Solomon Yim \\ School of Civil \& Construction Engineering \\ Oregon State University \\ Corvallis, Oregon 97331 USA
}

\begin{abstract}
This paper presents an accurate and efficient threedimensional computational model (3D numerical wave tank), based on fully nonlinear potential flow (FNPF) theory, and its extension to incorporate the motion of a laboratory snake piston wavemaker, to simulate experiments in a large-scale 3D wave basin (i.e. to conduct "virtual" or numerical experiments). The code is based on a higher-order boundary element method combined with a Fast Multipole Algorithm (FMA). Particular efforts were devoted to make the code efficient for large-scale simulations using high-performance computing platforms to complement experimental 3D wave basins. The numerical simulation capability can serve as an optimization tool at the experimental planning and detailed design stages. To date, waves that can be generated in the NWT include solitary, Cnoidal, and Airy waves. In this paper, we detail the model, mathematical formulation, and wave generation. Experimental or analytical comparisons with NWT results are provided for several cases to assess the accuracy and applicability of the numerical model to practical engineering problems.
\end{abstract}

\section{INTRODUCTION}

Over the past decade, as modern computing platforms gradually increased in power, accurate and efficient threedimensional (3D) computational wave-basins (called numerical wave tanks or simply NWTs in this paper) have been developed and refined, which simulate complex processes of ocean wave generation, propagation over arbitrary bottom topography, interaction with ocean structures, and dissipation over sloping beaches. These computational tools allow researchers to conduct "virtual" or numerical experiments. Until recently, fluid dynamic phenomena had been mostly investigated by performing laboratory experiments in large-scale 3D wave basins, which are both expensive and time consuming to operate. While not intended

\author{
Stephan Grilli \\ Department of Ocean Engineering \\ University of Rhode Island \\ Narragansett, Rhode Island 02882 USA
}

to replace such facilities, NWTs can be used to simulate and optimize planned physical experiments, ahead of time, and thus allow to more efficiently devote time and efforts to targeted laboratory experiments. As an added advantage, once validated, NWTs can calculate time series of detailed flow parameters (e.g., velocity, pressure) everywhere in the numerical model, while these are usually available only at a limited number of experimental probes (and at the sacrifice of flow-field intrusion), in laboratory experiments.

It is beyond the scope of this paper to provide an exhaustive literature review of the many methods that have been used to develop NWTs. We will only present and discuss a limited number of references, targeted to the type of models used in our work, i.e., models simulating nonlinear waves based on inviscid Fully Nonlinear Potential Flow (FNPF) theory, and implemented based on a higher-order Boundary Integral Equation (BIE) method, in a finite element (FEM) formalism, which is referred to as the Boundary Element Method (BEM). Besides its numerical efficiency and accuracy, the main advantage of the BEM in engineering applications is that the dimensionality of the discretized problem is reduced by one. Thus, 3D problems can be discretized using a surface-only (i.e. two-dimensional) mesh, which reduces the effort devoted to developing relevant numerical grids. Additionally, while the governing equation (here Laplace's equation) is satisfied only approximately over the 3D-BEM domain boundary, it is satisfied exactly within the domain. Due to the reduced dimensionality, the numerical solution can be computed efficiently even for higher-order schemes and highly resolved BEM surface grids. Hence, problems such as free-surface waves can be solved very accurately. Finally, if required, it is easier to regrid the (2-D) boundary mesh, unlike (3-D) domain-discretization based methods (e.g., FEM). This is particularly useful for moving boundary problems (e.g., free-surface waves), wherein regridding will be redistributing nodes evenly during wave 
propagation. The main drawback of the standard BEM, however, is that it yields non-symmetric and fully populated linear system matrices, which for large problems becomes prohibitive to solve, and thus require fast solution methods or a more advanced implementation that creates sparse matrices. This aspect, of improving the numerical efficiency of a 3DBEM-NWT, is one of the main aspects of this paper that is extensively discussed later.

Historically, the BEM has been studied from the perspectives of classical boundary integral equations, mathematical analysis, and engineering applications (see, e.g., $[1,2])$. Following recent developments and advances in High Performance Computing (HPC), however, the BEM is receiving a renewed attention from various research communities resulting in new interesting practical developments and applications of the methodology. The solution technique used, the Fast Multipole Algorithm (FMA; see, e.g., [3-5]), makes the 3D-BEM much more efficient when using large-size discretizations required when solving practical engineering problems (which may require $O\left(10^{5}\right.$ to $10^{6}$ ) BEM nodes). The FMA is an important and relatively recent development (particularly in the context of NWTs) that has brought the BEM to the forefront of numerical methods used in such problems. The principle of the FMA is first to approximate the free space Green's function of the problem governing equations by a series of spherical harmonics. Then, for each BEM discretization node, a hierarchy of increasingly distant sub-domains is defined, in which the full Green's function is used only in the nearest sub-domains, and a decreasing number of harmonics are used to represent the Green's function (down to no harmonics), for increasingly distant sub-domains. Doing so both accelerates the computation (through numerical integration) of the non-zero coefficients in the BEM algebraic system matrices and creates large empty blocks in such matrices for sub-domains beyond a cut-off distance, thus yielding a sparse structure. When properly implemented, a BEM-FMA code can achieve an $N$ $\log N$ numerical complexity (with $N$ the boundary discretization size). Reference [4] reports on the initial (scalar) implementation and application of the FMA method in the 3DNWT used in this work.

\section{BACKGROUND OF FNPF THEORY AND MODELING OF FREE-SURFACE WAVES}

Potential flow theory solves inviscid, incompressible, Euler equations for irrotational flows. The governing equation for potential flows is mass conservation, which is expressed as a Laplace's equation for the potential, i.e., a second-order linear elliptic partial differential equation. Nonlinearity in wave processes originates from the presence of, and equations governing, the free surface, i.e., the dynamic (DFSBC) and kinematic (KFSBC) free surface boundary conditions. When full nonlinearity is kept in the latter, this yields FNPF equations.

Earlier work in applying FNPF theory to modeling various strongly nonlinear nearshore waves [6,7] indicates that it is accurate outside of the surfzone, up to the breaking point, where viscous effects are usually negligible, and hence vorticity is not generated, except in thin bottom and freesurface boundary layers. Additionally, in the presence of submerged or floating ocean structures, viscous effects are also negligible for large-scale bodies (such as ships); for small bodies (e.g., pipelines), while viscosity may be locally important for the flow around the structure, it will typically be negligible for the large scale (or far-field) wave flow itself. The assumption of incompressible fluid is valid when there is no air-water mixing (i.e., no bubbles), which is mostly the case for non-breaking waves. Accordingly, most results of classical wave dynamics and applications to date have been based on FNPF theory, or on other equations derived from it through perturbation expansions (e.g., Stokes waves [8]; Boussinesq equations; see, e.g., [9]). One severe limitation of FNPF theory, however, is that wave overturning and breaking will cause flow (i.e., breaking jet) penetration, which violates the governing equations and thus interrupts computations based on this flow model. Hence, in FNPF-NWTs, numerical absorbing beaches have been developed and used to prevent waves from overturning, through the absorption of the energy of steeper waves, usually by specifying an "absorbing" pressure distribution on the free surface.

In the 2D-FNPF models (and NWTs) initially developed [10-15], both the solution method (based on a direct Gaussian elimination scheme) and the computation of the matrix elements limited the size of problems that could be handled. With regard to fluid-structure interactions, using 2DFNPF theory, Lin [10] studied the nonlinear behavior of the flow near the intersection point of a free surface and a floating body (represented by a piston wavemaker). A numerical algorithm was proposed to accommodate the singularity resulting from satisfying conflicting boundary conditions near the intersection point. This aspect was revisited by Grilli, et al $[11,12]$ in the context of a different BEM model implementation, which used double nodes with specific continuity and compatibility conditions, and extended to the generation of waves by paddle flap or piston wavemakers [11$15]$.

As part of a pioneering project on 3D numerical simulations of nonlinear water waves, Zandbergen and coworkers [16] generated substantial research based on using the BEM and FNPF approaches. This work confirmed that, for large objects with characteristic dimensions on the order of one wavelength, viscous as well as compressibility effects can be neglected in the fluid flow: while the assumption that the flow is potential is not valid at all stages of physical processes, it is valid for many practical engineering situations. Liu et al. [17] compared BIE solutions of 2D nonlinear water wave problems using free space or periodic Green's functions. Cooker [18] further develops the method for 2D nonlinear wave propagation over irregular beds.

In a series of research papers that represents the basis for the present work [19-21], Grilli and co-workers developed 
an accurate and versatile 3D-NWT based on FNPF theory, which directly extended their earlier 2D work (e.g., [7,11-15]). This 3D-NWT was initially based on combining a $3^{\text {rd }}$-order BEM, based on a free-space Green's function in the physical space, with an explicit time updating based on $2^{\text {nd }}$-order Taylor series expansions, in a Mixed Eulerian-Lagrangian (MEL) formulation [19-21]. To improve the computational efficiency, the method was later extended [4,21-24] to using a Fast Multipole Algorithm (FMA). The method used for solving the BEM algebraic system was Generalized Minimal Residual (GMRES). In applications of the BEM-FMA model, the numerical complexity was found to be nearly proportional to the problem size $N$.

Despite its demonstrated success for academic-type applications, the range of engineering applications that could practically be solved using Grilli et al.'s 3D-NWT, however, was limited by the size of problems that could be solved on a single processor machine. This was because both the FMA and the BEM had been implemented in scalar mode in the original model. Also, while wavemakers had been used in a few applications (e.g., [19-21]) the FMA implementation of the 3D-NWT did not feature a snake piston wavemaker for simulating laboratory wave generation (e.g., such as in Oregon State University's (OSU) 3D wave basin), which is an important requirement to be able to model shallow water wave in a physical wave basin, nor was there a fully operational absorbing beach (AB), as used in 2D applications.

In this work, we report on recent improvements of the 3D-NWT implementation and validation by: (i) addition of a snake piston wavemaker for arbitrary wave generation (with the development of a wave generation module based on the control software driving the actual wavemaker at OSU's wave basin); and (ii) an efficient absorbing beach. These improvements bring the 3D-NWT model to the level of an engineering tool, able to simulate meaningful laboratory experiments in a large-scale wave basin, on a moderate size HPC platform. This is demonstrated by comparing numerical results for $3 \mathrm{D}$ wave generation and propagation to laboratory measurements.

In the following, we first present a summary of the mathematical model and numerical algorithms underlying the FNPF 3D-NWT, and then we describe the enhancements implemented into the NWT and their systematic validation for the generation of solitary waves and periodic waves (Cnoidal and Airy waves). Applications are presented, with a comparison of numerical results to experimental data and theoretical solutions.

\section{MATHEMATICAL MODEL}

As indicated before, under FNPF theory, the governing differential equation, i.e., mass conservation, is governed by the Laplace equation. With $\phi$ denoting the velocity potential and the velocity given by $\mathbf{u}=\nabla \phi=(u, v, w)$, this reads,

$$
\nabla^{2} \phi=\frac{\partial^{2} \phi}{\partial x^{2}}+\frac{\partial^{2} \phi}{\partial y^{2}}+\frac{\partial^{2} \phi}{\partial z^{2}}=0
$$

Figure 1 shows the general set-up and typical geometry of the 3D-NWT, in the case of wave generation by a snake piston wavemaker on the leftward boundary $\Gamma_{\mathrm{w}}$, with stroke function $x_{p}(y, t)$. Wave elevation $\eta(x, y)$ on the free surface boundary $\Gamma_{\mathrm{f}}$ is defined with respect to the still water level, corresponding to the $(x, y)$ plane (that is $z=0)$. The tank axis is at $y=0$, and $x$ is positive rightward, in the initial direction of wave propagation away from the wavemaker. As in OSU's wave basin, we assume here that the four sides of the tank are vertical boundaries, one of these being the moving snake wavemaker and the opposite end, parallel to the $(y, z)$ plane, a moving absorbing piston boundary $\Gamma_{\mathrm{ap}}$. The two sidewalls $\Gamma_{\mathrm{s}}$, parallel to the $(x, z)$ plane, are fixed in location. The impermeable bottom boundary $\Gamma_{\mathrm{b}}$ is represented in the figure with a constant depth $h_{0}$, but can be specified to be sloping or with an arbitrary topography.

On the free surface $\Gamma_{\mathrm{f}}$, the potential satisfies the nonlinear kinematic (KFSBC) and dynamic (DFSBC) boundary conditions,

$$
\begin{aligned}
& \frac{D \mathbf{R}}{D t}=\mathbf{u}=\nabla \phi \\
& \frac{D \phi}{D t}=-g z+\frac{1}{2} \nabla \phi . \nabla \phi
\end{aligned}
$$

respectively, with $\boldsymbol{R}(t)$ the position vector of a fluid particle on the free surface and $g$ the acceleration due to gravity. Both conditions are required since there are two unknowns (position and potential) on the free surface. The KFSBC states that the normal velocity is equal to the normal fluid velocity at the surface, when following a fluid particle at the free surface (this means that such particles remain on the free surface). The DFSBC, obtained from Bernoulli's equation (i.e., an integration of Euler equations), states that the pressure on the free surface equals the atmospheric pressure, which is assumed here to be zero for simplicity.

For fixed boundaries, a no-flow condition (zero flux) is specified as $\frac{\partial \phi}{\partial n}=0$, where $\mathbf{n}$ is the normal vector to the surface (pointing outside the fluid).

For a moving (piston) wavemaker boundary, both the motion (stroke function) and velocity are prescribed based on waves to be generated, by way of a wavemaker theory, as,

$$
x=x_{p}(y, t) ; \frac{\partial \phi}{\partial n}=\mathbf{u}_{p} . \mathbf{n}
$$

where $x_{p}$ and $\mathbf{u}_{\mathrm{p}}$ are the wavemaker stroke and velocity, respectively.

The initial free surface boundary condition (at $t=0$ ) is given by specifying a cold start in the NWT, with a still water level $(z=0)$ and zero potential (Dirichlet BC), and all other surfaces having zero or specified normal fluxes (Neumann BC). 


\section{NUMERICAL IMPLEMENTATION}

A higher-order BEM is used to solve Eq.(1), whereby, Green's second identity is applied to transform Laplace's equation into a BIE, which is discretized on the boundary. The numerical implementation is summarized below. For details, see [1920,23].

4.1 Governing equation

The BIE representation of Eq.(1) reads,

$\alpha\left(\mathbf{x}_{l}\right) \phi\left(\mathbf{x}_{l}\right)=\int_{\Gamma(t)}\left\{\frac{\partial \phi}{\partial n}(\mathbf{x}) G\left(\mathbf{x}, \mathbf{x}_{l}\right)-\phi(\mathbf{x}) \frac{\partial G}{\partial n}\left(\mathbf{x}, \mathbf{x}_{l}\right)\right\} d \Gamma$

where $\Gamma$ denotes the boundary of the fluid domain, $\alpha\left(\mathbf{x}_{l}\right)$ is function of the exterior solid angle made by the boundary at the collocation point $\mathbf{x}_{l}$, and the 3D free space Green's function for Laplace's equation is defined as,

$$
G\left(\mathbf{x}, \mathbf{x}_{l}\right)=\frac{1}{4 \pi r}
$$

where $r=\left|\mathbf{x}-\mathbf{x}_{l}\right|$ is the distance from the source point $\mathbf{x}$ to the collocation point $\mathbf{x}_{l}$ (both on the boundary). The normal derivative of the Green’s function further reads,

$$
\frac{\partial G}{\partial n}\left(\mathbf{x}, \mathbf{x}_{l}\right)=\nabla G \cdot \mathbf{n}=-\frac{1}{4 \pi} \frac{\mathbf{r} \cdot \mathbf{n}}{r^{3}}
$$

\subsection{Time integration}

Second-order explicit Taylor series expansions are expressed for the free surface position and potential, updated to the next time step $t+\Delta t$, as a function of the solution at $t$,

$$
\begin{aligned}
& \mathbf{R}(t+\Delta t)=\mathbf{R}(t)+\Delta t \frac{D \mathbf{R}}{D t}(t)+\frac{(\Delta t)^{2}}{2} \frac{D^{2} \mathbf{R}}{D t^{2}}(t)+O\left\{(\Delta t)^{3}\right\} \\
& \phi(t+\Delta t)=\phi(t)+\Delta t \frac{D \phi}{D t}(t)+\frac{(\Delta t)^{2}}{2} \frac{D^{2} \phi}{D t^{2}}(t)+O\left\{(\Delta t)^{3}\right\}
\end{aligned}
$$

These expressions yield an explicit, stable, and efficient MEL time stepping scheme. In these equations, zero ${ }^{\text {th }}$-order coefficients are given by the free-surface geometry and potential at time $t$. First-order coefficients are evaluated from the free surface BCs Eqs.(2,3), also as a function of geometry and the BIE solution for $\phi$ and $\partial \phi / \partial$ at time $t$. Based on Eqs.(2,3), second-order coefficients are expressed as,

$$
\begin{aligned}
& \frac{D^{2} \mathbf{R}}{D t^{2}}=\frac{D \mathbf{u}}{D t}=\frac{\partial \mathbf{u}}{\partial}+\mathbf{u} \cdot \nabla \mathbf{u}=\nabla \frac{\partial \phi}{\partial t}+\nabla \phi \cdot \nabla(\nabla \phi) \\
& \frac{D^{2} \phi}{D t^{2}}=-g \frac{D z}{D t}+\frac{1}{2} \frac{D(\nabla \phi \cdot \nabla \phi)}{D t}=-g w+\mathbf{u} \cdot \frac{D \mathbf{u}}{D t}
\end{aligned}
$$

The expressions in Eq.(9) can be calculated as a function of geometry and the BIE solutions for both the potential and the time derivative of the potential at time $t$ (i.e., $\partial \phi / \partial$ and $\gamma^{2} \phi / \partial \alpha$ ). It should be emphasized that this second BIE solution uses the same system matrix as the first one and has boundary conditions, which can be calculated as a function of the solution of the first BIE.

\section{SALIENT FEATURES OF THE NUMERICAL ALGORITHMS}

Salient features of the numerical algorithms combining higherorder BIE solutions and an explicit time updating scheme can be summarized as follows (as above, see [19,20,23] for details):

- Second-order Taylor series coefficients, used in the time updating, are obtained from time derivatives of the boundary potential and flux, which are obtained from solving another BIE. Since this is performed using the same geometry as that for the first BIE for the potential, the same discretized BEM algebraic system matrix is used, with a different right hand side; hence the solution of the second BIE comes only at a moderate additional time cost.

- The time step is adapted as a function of the minimum distance between two nodes on the free surface, based on a constant mesh Courant number C0 0.45 [12].

- The numerical solution of the algebraic BEM systems uses GMRES, wherein the matrix vector products are replaced by the fast multipole algorithm (FMA) for distant sources points, relative to a given collocation node $[4,23]$. This algorithm, which uses multipole expansions and tree data structures, avoids the full assembling of the discretized system matrix in memory. The theoretical computational complexity of the FMA is $\mathrm{O}(\mathrm{N} \log \mathrm{N})$, where $\mathrm{N}$ is the number of nodes on the boundary [4,22]. This is a very good improvement over the standard GMRES implementation, which results in an O(N2) performance. More details on the FMA implementation can be found in [4]. Here, the FMA was modified to allow for a parallel implementation on computer systems with multi-core nodes that share a large central memory.

- The majority of the 3D-NWT code was developed in FORTRAN, while the FMA algorithms used libraries written in C language.

- The user's input to the 3D-NWT was designed to be minimal, in the form of the broad dimensions of the NWT and number of elements in each direction, plus a few other control and FMA parameters. The 3D surface mesh is automatically generated in the model, based on input parameters, thus resulting in a considerable time saving for the user.

- A node regridding technique can be automatically applied for any user-specified iterations so that free surface nodes are redistributed evenly over the grid. This option helps prevent inaccuracies and 
instabilities due to a very uneven distribution of nodes during wave propagation (which may occur due to Stokes drift for strongly nonlinear waves).

\section{PISTON WAVEMAKER MOTION AND WAVE GENERATION}

Boundary conditions for a plane (2D) piston wavemaker were derived in [13], for generating solitary, Cnoidal, or other types of elementary waves. Grilli, et al. [24] extended this 2D wavemaker generation to arbitrary irregular waves, based on a target wave energy spectrum (such as a JONSWAP spectrum), and the possibility to correct the wavemaker stroke function for reflection in the NWT. In the 3D-NWT, Brandini and Grilli [19] implemented a snake flap wavemaker to model 3D wave focusing (see also, [21]).

Here, to more accurately generate shallow water waves, a snake piston wavemaker similar to that at OSU's wave basin is implemented in the 3D-NWT. To ensure a match between experimental and numerical wave generation, the wavemaker paddle stroke function in Eq. (4) is specified such as to closely approximate the laws of motion of the mechanical actuators used in OSU's basin. In the latter, the multidirectional (or snake) wavemaker has 29 rigid segments connected at the edges to 30 independently controlled actuators. While, for the purpose of validating the new 3DNWT implementation, we will only present in this paper applications with long-crested 2D waves, more general and complex cases of wave generation will be reported in future work.

The wavemaker stroke were general functions of time $t$ and lateral position $y$ (there is no vertical variation for a piston wavemaker), with stroke $x_{p}(y, t)$ (Fig. 1), velocity: $\left|\mathbf{u}_{\mathrm{p}}\right|=$ $u_{p}=d x_{p} / d t$, and acceleration $a_{p}=d u_{p} / d t$, so that an arbitrary snaking motion could be specified.

A new module (Wavegen) was implemented to generate the various stroke functions $\left(x_{p}, u_{p}, a_{p}\right)$ required to simulate laboratory experiments in OSU's wave basin, for various type of standard waves, based on algorithms used in OSU's wavemaker driver software. At present, Wavegen offers three types of long-crested wave generation capabilities, which will be illustrated in the present applications: solitary, Cnoidal, and Airy waves. Hughes [25] presented various issues related to wave generation using wavemakers, including more complex or nonlinear waves.

\section{VERIFICATION OF GENERATION, PROPAGATION AND ABSORPTION OF SIMPLE WAVES IN THE NWT}

The original model, on which this NWT is based, has already been validated for a number of theoretical applications (e.g., for solitary waves) where both convergence and accuracy of the BEM solution were assessed as a function of mesh and time step parameters [22]. None of these earlier validation tests, however, had been conducted for large 3D grids and with the solution performed using the new parallelized FMA algorithm. Additionally, although the equivalent 2D-FNPF model had been validated for strongly nonlinear waves using experimental data [15], no such comparison of 3D-NWT results with detailed laboratory experiments in a large 3D wave tank, such as available at OSU, had been done to date.

In this section, we perform numerical simulations to verify that the new implementation of wave generation by a piston wavemaker, propagation over constant depth, and energy absorption by the “absorbing beach", perform as expected. In these validation applications, only simple longcrested (i.e., 2D) waves are generated, as detailed in the previous section, and, hence, the piston wavemaker moves as a whole (i.e., there is no snaking effect). For such cases, the width of the NWT does not really matter and can be small, to save on computational time; we thus are dealing here with a narrow 3D-NWT. In the following computations, the 3DNWT has a length of $25 \mathrm{~m}$, a depth of $h=0.75 \mathrm{~m}$, and a width of $1 \mathrm{~m}$ for solitary waves, or $0.5 \mathrm{~m}$, for Cnoidal, or Airy waves. These waves are generated at the wavemaker, propagate in the tank and are absorbed at the far end in an Absorbing Beach (AB) of $3 \mathrm{~m}$ length (with an AB coefficient $v_{o}=400 \mathrm{~kg} /(\mathrm{m} . \mathrm{s})$; see [14]). Although OSU's wave basin has larger dimensions (length $48.8 \mathrm{~m}$ and width $26.5 \mathrm{~m}$ ), the reduced dimensions (along length and width) in the model are acceptable, as already indicated, since we are only generating $2 \mathrm{D}$ waves (in the vertical plane). Results detailed below include a comparison with experiments and theoretical wave profiles.

\subsection{Solitary waves}

In this simulation, the BEM grid has 200 elements along the length, 5 elements along the width, and 8 elements along the depth. This yields a total of $N=6,138$ nodes and $M$ $=5,280$ elements. Two waves are generated, with targeted heights $H=0.3$ and $0.45 \mathrm{~m}$ in depth $h=0.75 \mathrm{~m}$, or $H / h=0.4$ and 0.6, respectively (Fig. 3); hence these are strongly nonlinear waves. For both waves, numerical wave profiles, at the wavemaker and 3 wave gages, and particle velocities at the third gage (at a depth $z=-0.61 \mathrm{~m}$ ), are found to agree well with experimental results. Wave absorption (absorbing piston and absorbing pressure beach) at the end of the tank appears to be working well as no significant reflected waves can be seen in the wave gage records.

\subsection{Cnoidal waves}

Here, the NWT-BEM grid has 200 elements along the length, 5 elements along the width, and 5 elements along depth. This yields a total number of nodes $N=4,896$ and elements $M=4,050$. The targeted wave height is $H=0.3 \mathrm{~m}$ and period $T=3.5 \mathrm{~s}$; the expected wavelength from Cnoidal theory is $L=9.84$; hence, $L / h=13.1$, which corresponds to a fairly long (but still intermediate depth) wave, and $H / h=0.4$, indicating a strongly nonlinear wave. Additionally, Cnoidal theory predicts a trough depth of $-0.08 \mathrm{~m}$ and a crest height of $0.22 \mathrm{~m}$ (Fig. 6). Fig. 6 shows that the initial numerical wave profile at the wavemaker agrees well with the theoretical one. Fig. 7 shows wave profiles computed at a few gages, and Fig. 8 shows particle velocity components at one location. The good periodicity of these parameters, once simulations have 
reached a quasi-steady state, indicates that wave absorption is working adequately in the NWT. Note, in Fig. 7, while both crest height and trough depth stay quite close to the expected values, the small oscillations in each wave trough, which are a well-known indication of the generation of higher-order harmonics in the generation of strongly nonlinear waves using a low-order wave theory.

\subsection{Airy Waves}

The grid used here is the same as for Cnoidal waves. The wave height is $H=0.2 \mathrm{~m}$ and period $T=3.0 \mathrm{~s}$, yielding a linear wavelength $L=7.68 \mathrm{~m}$ (Eq. A13); hence, $L / h=10.2$, which corresponds to an intermediate wave, and $H / h=0.27$ and $H / L=0.026$, indicative of a fairly strongly nonlinear wave. In Fig. 9, we see, the initial wave shape at the wavemaker is, as expected, closely sinusoidal and trough/crest symmetric. Fig. 10, however, shows that, as expected, the linear wave gradually adjusts its shape, to reach a permanent form profile consistent with wave nonlinearity. Thus, at the farther gage down the NWT (g3), wave profiles have become fairly steady, with narrower and taller crests (at $0.15 \mathrm{~m}$ ) and wider and shallower troughs (at $-0.06 \mathrm{~m}$ ). Wave particle velocities at locations far down the tank also become quasisteady (Fig. 11). Wave absorption in the absorbing beach is thus also working well in this case.

\section{CONCLUSIONS}

This paper presented improvements in the implementation of an existing computation model (3D-NWT), based on FNPF theory, to make it an efficient tool to complement experimental facilities such as OSU's 3D wave basin or elsewhere. The numerical algorithms and mathematical formulations involved in furthering the development of wave generation capabilities (i.e., for solitary, Cnoidal and Airy waves) are provided. Comparisons with experiments for simple waves illustrate that numerical and experimental results are in good agreement and provide a strong basis for the use of the 3D-NWT code for all appropriate and practical engineering purposes. More complex cases of wave generation, for fully $3 \mathrm{D}$ waves and/or varying bathymetry in the NWT, will be reported in future work.

\section{ACKNOWLEDGEMENTS}

The first two authors are thankful to the Office of Naval Research ONR-N00014-11-1-0094 and the Department of Energy DOE-G0107G for the financial support that made this work possible.

\section{REFERENCES}

[1] Brebbia, C.A., Telles, J.C.F., and Wrobel, L.C. Boundary element techniques: theory and applications in engineering. Springer-Verlag, Berlin, 1984.

[2] Hsiao, G.C., and Wendland, W.L. Boundary integral equations. Springer-Verlag, Berlin, 2008.

[3] Nishimura, N. Fast multipole accelerated boundary integral equation methods. Appl. Mech. Rev.; 55(4): 299-324, 2002.
[4] Fochesato, C., and Dias, F. A fast method for nonlinear three-dimensional free-surface waves. Proc. Royal Soc.; A 462: 2715-2735, 2006.

[5] Chaillat, S., Bonnet, M., and Semblat, J-F. A multi-level fast multipole BEM for 3-D elastodynamics in the frequency domain. Comp. Methods in Appl. Mech. and Engng.; 197(4950): 4233-4249, 2008.

[6] Dommermuth, D.G., Yue, D.K.P., Lin, W.M., and Rapp, R.J. Deep-water plunging breakers: a comparison between potential theory and experiments. J. Fluid Mech.; 189: 423442, 1988.

[7] Grilli, S.T., and Horrillo, J. Shoaling of periodic waves over barred-beaches in a fully nonlinear numerical wave tank. Intl. J. Offshore and Polar Engng,; 9(4): 257-263, 1999.

[8] Dean, R.G. and Dalrymple, R.A. Water wave mechanics for engineers and scientists. World Scientific Publishing, 2000.

[9] Wei, J., Kirby, J.T., Grilli, S.T. and Subramanya, R. A Fully Nonlinear Boussinesq Model for Surface Waves. Part1. Highly Nonlinear Unsteady Waves. J. Fluid Mech.; 294: 7192, 1995.

[10] Lin, W.M., 1984, "Nonlinear motion of the free surface near a moving body," $\mathrm{PhD}$ thesis, Department of Ocean Engineering, Massachusetts Institute of Technology.

[11] Grilli, S., and Svendsen, I.A. Corner problems and global accuracy in the boundary element solution of nonlinear wave flows," Engng. Anal. Boundary Elements; 7(4): 178195, 1990.

[12] Grilli, S.T., and Subramanya, R. Numerical modeling of wave breaking induced by fixed or moving boundaries. Comput. Mech.; 17: 374-391, 1996.

[13] Grilli, S.T. Fully nonlinear potential flow models used for long wave runup prediction, in Long-Wave Runup Models (H. Yeh, P. Liu, and C. Synolakis, eds.), World Scientific Publishing, Singapore, pp.116-180, 1997.

[14] Grilli, S.T., and Horrillo, J. Numerical Generation and Absorption of Fully Nonlinear Periodic Waves. J. Engng. Mech.; 123 (10): 1060-1069, 1997.

[15] Grilli, S.T., Voropayev, S., Testik, F.Y., and Fernando, H.J.S. Numerical Modeling and Experiments of Wave Shoaling over Buried Cylinders in Sandy Bottom, in Proc. 13th Offshore and Polar Engineering Conference (ISOPE03, Honolulu, USA, May 2003), pp. 405-412, 2003.

[16] Berkvens, P.J.F. Floating bodies interacting with water waves- development of a time-domain panel method. Zandbergen, P.J., (advisor), PhD thesis, University of Twente, Netherland, 1998.

[17] Liu, P.L.F., and Hsu, H.W. Applications of boundary integral equation methods for two-dimensional non-linear water wave problems," Intl. J. Num. Meth. in Fluids; 15: 1119-1141, 1992.

[18] Cooker, M.J. A boundary-integral method for water wave motion over irregular beds. Engng. Anal. Boundary Elements; 7(4): 205-213, 1990.

[19] Brandini, C., and Grilli, S.T. Modeling of freak wave generation in a 3D-NWT, in Proc. 11th Offshore and Polar 
Engng. Conf. (ISOPE01, Stavanger, Norway, June 2001), Vol III: 124-131, 2001.

[20] Grilli, S.T., Vogelmann, S., and Watts, P. Development of a 3D numerical wave tank for modeling tsunami generation by underwater landslides. Engng. Anal. Boundary Elements; 26(4): 301-313, 2002.

[21] Fochesato, C., Grilli, S.T., and Dias F. Numerical modeling of extreme rogue waves generated by directional energy focusing. Wave Motion; 44: 395-416, 2007.

[22] Sung, H.G., and Grilli, S.T. BEM Computations of 3D Fully Nonlinear Free Surface Flows Caused by Advancing Surface Disturbances. Intl. J. Offshore and Polar Engng.; 18(4): 292-301, 2008.

[23] Grilli, S.T., Dias, F., Guyenne, P., Fochesato, C., and Enet, F. Progress In Fully Nonlinear Potential Flow Modeling Of 3D Extreme Ocean Waves, Chapter 3 in Advances in Numerical Simulation of Nonlinear Water Wave (Q. Ma, ed.),Vol. 11 in Series in Advances in Coastal and Ocean Engineering, World Scientific Publishing Co. Pte. Ltd., pp. 75-128, 2010.

[24] Grilli, S.T., Harris, J., and Greene, N. Modeling of Wave-induced Sediment Transport around Obstacles. In Proc. 31st Intl. Coastal Engng. Conf. (J. McKee Smith, ed.) (ICCE08, Hamburg, Germany, September, 2008), World Scientific Publishing Co. Pte. Ltd, pp.1,638-1,650, 2009.

[25] Steven A. Hughes. Physical models and laboratory techniques in coastal engineering. World Scientific Publishing, 1993.

\section{Figures}

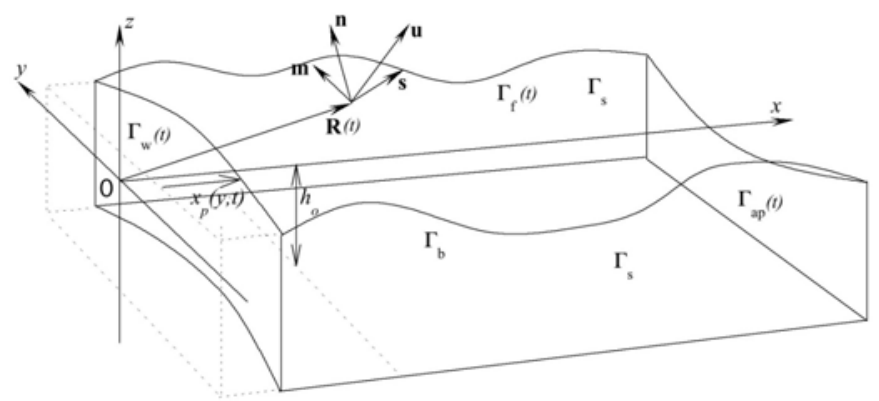

Fig. 1: Sketch of 3D-NWT geometry and parameters, for wave generation by a snake piston wavemaker (notation and details of mathematical model can be found in section 3)

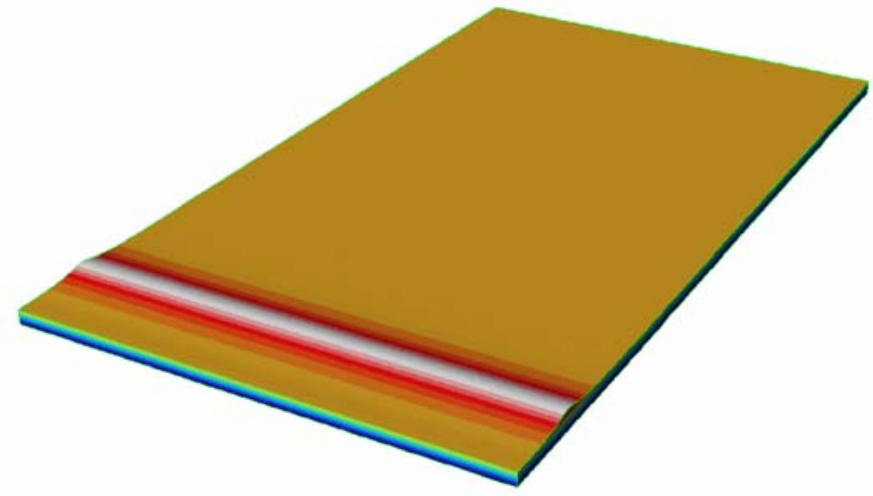

Fig. 2: Snapshot of 3D-NWT simulaitons for the propagation of a solitary wave over constant depth in a geometry identical to that of OSU's wave basin (48.8 m long, $26.5 \mathrm{~m}$ wide, 0.78 $\mathrm{m}$ deep).

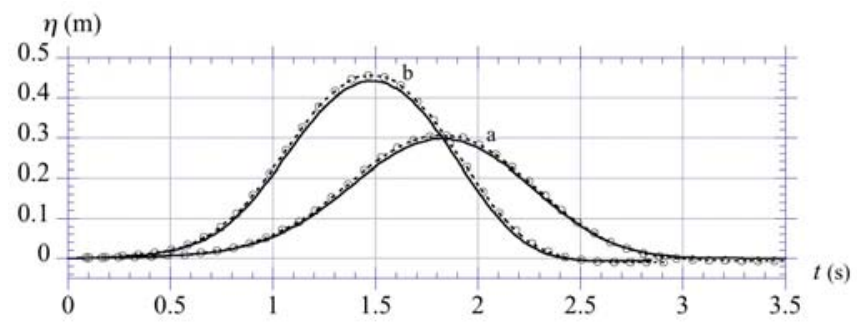

Fig. 3: Solitary waves of target height $H=$ (a) $0.3 \mathrm{~m}$; (b) 0.45 $\mathrm{m}$, in water depth $h=0.75 \mathrm{~m}$. Surface elevations versus time at the wavemaker, in: (-) numerical model; (-o-) OSU's 3D tank experiments (experimental data were shifted by a $0.04 \mathrm{~s}$ time lag; only $25 \%$ of experimental points are shown).
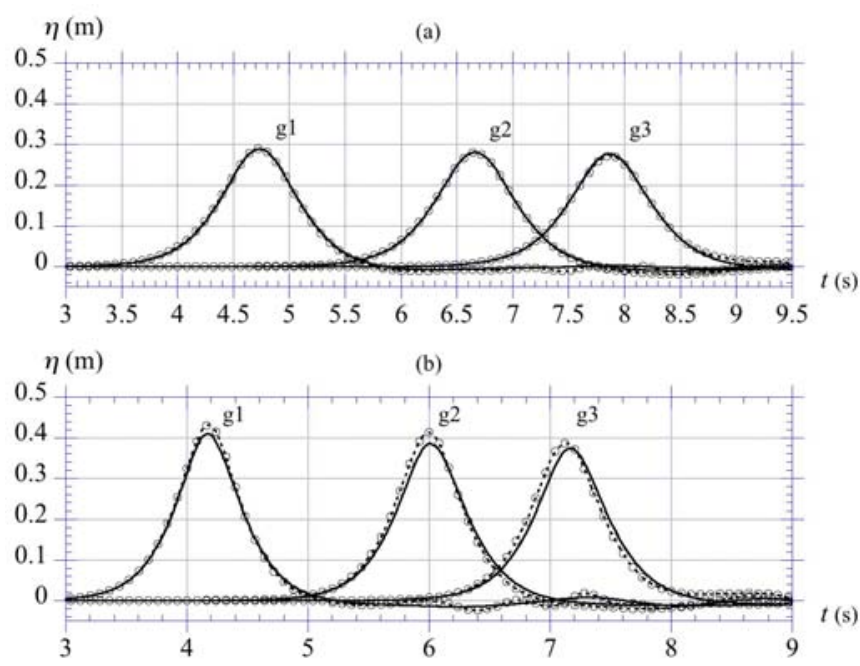

Fig. 4: Solitary waves of target wave height $H=$ (a) $0.3 \mathrm{~m}$; (b) $0.45 \mathrm{~m}$, in depth $h=0.75 \mathrm{~m}$. Numerical (-) and experimental (-o-) surface elevations as functions of time, at three gages at $x$ $=8.8 \mathrm{~m}$ (g1), $14.9 \mathrm{~m}$ (g2), and $18.7 \mathrm{~m}$ (g3), with $y=0$

(experimental data were shifted by a $0.16 \mathrm{~s}$ time lag; only $25 \%$ of experimental points are shown). 

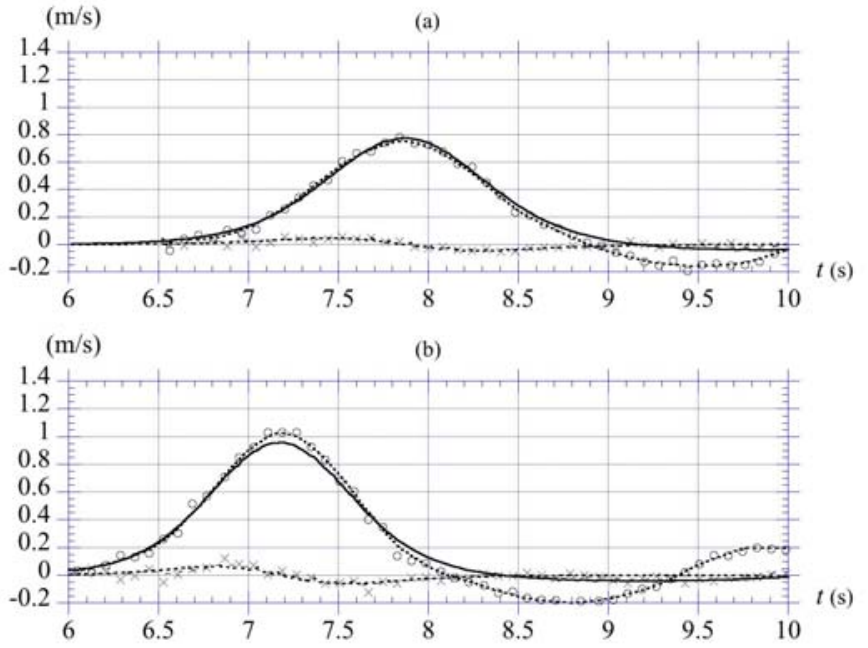

Fig. 5: Solitary waves of target wave height $H=$ (a) $0.3 \mathrm{~m}$; (b) $0.45 \mathrm{~m}$, in depth $h=0.75 \mathrm{~m}$. Numerical $(-)$ and experimental (-o-) water particle velocity components $(u, w)$ as functions of time, at gage g3 location: $x=18.7 \mathrm{~m}, y=0$, at depth $z=-$ $0.61 \mathrm{~m}$ (only $25 \%$ of experimental points are shown).

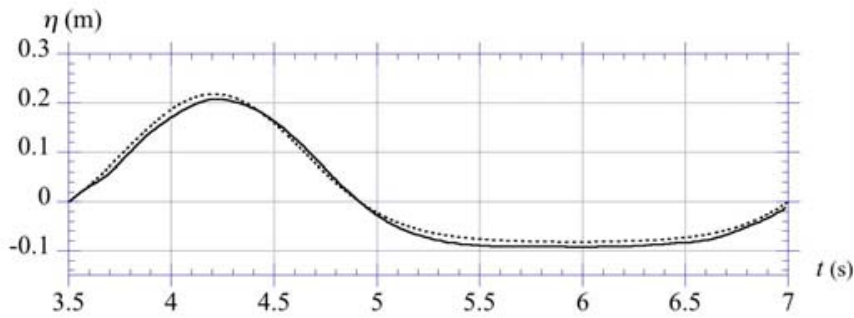

Fig. 6: Cnoidal wave of target height $H=0.3 \mathrm{~m}$ and period $T$ $=3.5 \mathrm{~s}$, in water depth $h=0.75 \mathrm{~m}$. Numerical $(-)$ and theoretical (- - -) surface elevations at the wavemaker as functions of time.

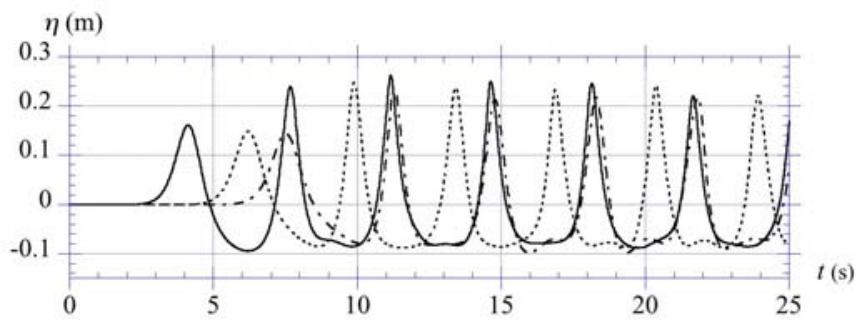

Fig. 7: Cnoidal wave of Fig. 6. Numerical wave elevation as a function of time, at three wave gages at $x=(\mathrm{g} 1:-) 8.8 \mathrm{~m}$, (g2: - - -), $14.9 \mathrm{~m}$ and (g3: - .) $18.7 \mathrm{~m}$, with $y=0$.

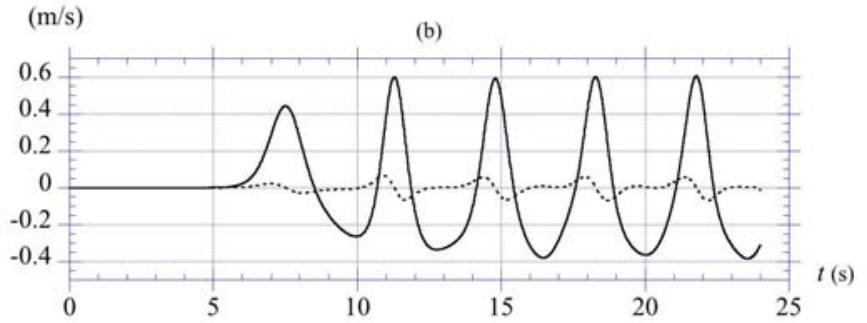

Fig. 8 : Cnoidal wave of Figs. 6,7. Numerical: wave particle velocity components $u(-), w(--)$ as functions of time at gage g3, with $x=18.7 \mathrm{~m}, y=0$, at depth $z=-0.61 \mathrm{~m}$.

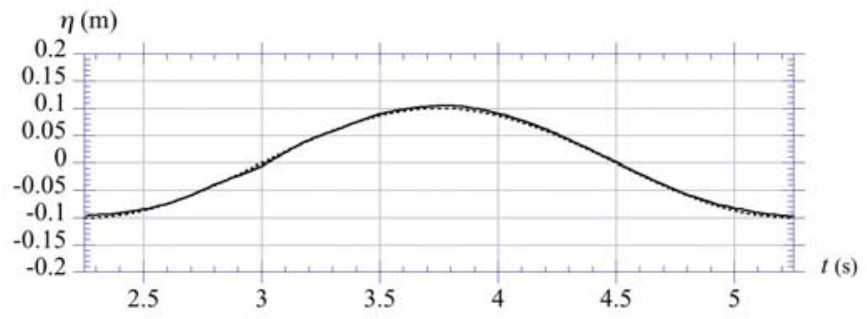

Fig. 9: Airy wave of target height $H=0.2 \mathrm{~m}$ and period $T=$ $3.0 \mathrm{~s}$, in water depth $h=0.75 \mathrm{~m}$. Numerical $(-)$ and theoretical (- - -) surface elevations at the wavemaker as functions of time.

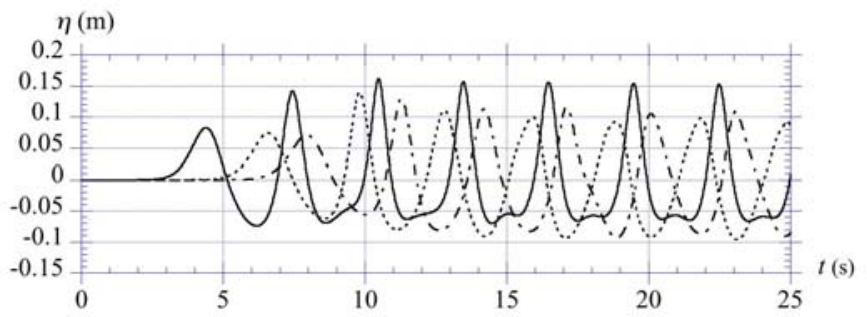

Fig. 10: Airy wave of Fig. 9. Numerical surface elevation as a function of time, at three wave gages at $x=(\mathrm{g} 1:-) 8.8 \mathrm{~m}$, (g2: - - ), $14.9 \mathrm{~m}$ and (g3: — .) $18.7 \mathrm{~m}$, with $y=0$.

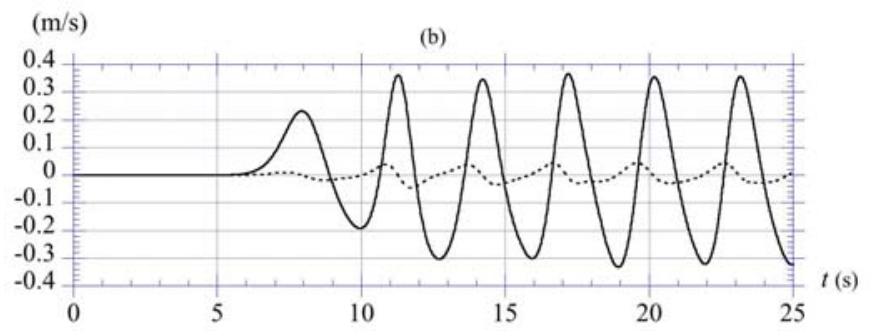

Fig. 11: Case of Figs. 9,10, numerical: wave particle velocity components $u(-), w(--)$ as functions of time at $x=18.7 \mathrm{~m}$, $z=-0.61 \mathrm{~m}, y=0$. 